\title{
Optical qubit generation by state truncation using an experimentally feasible scheme
}

\author{
ŞAHIN KAYA ÖZDEMIR †' \\ MASATO KOASHI ${ }^{\dagger}$, and NOBUYUKI IMOTO ${ }^{\dagger} \|$ \\ † CREST Research Team for Interacting Carrier Electronics, School of \\ Advanced Sciences, Graduate University for Advanced Studies (SOKEN), \\ Hayama, Kanagawa 240-0193, Japan \\ $\S$ Nonlinear Optics Division, Institute of Physics, Adam Mickiewicz University, \\ 61-614 Poznań, Poland \\ || NTT Basic Research Laboratories, 3-1 Morinosato Wakamiya, Atsugi, \\ Kanagawa 243-0198, Japan
}

\begin{abstract}
Generation of arbitrary superposition of vacuum and one-photon states using quantum scissors device (QSD) is studied. The device allows the preparation of states by truncating an input coherent light. Optimum values of the intensity of the coherent light for the generation of any desired state using the experimentally feasible QSD scheme are found.
\end{abstract}

\section{Introduction}

In recent years, many experiments falling into the quantum domain of optical fields have been performed in optics laboratories. The field has received much attention motivated not only by the excitement of studying the fundamentals of quantum mechanics, but also by the potential use of quantum optical light for the manipulation and transmission of information. Consequently, the quantum engineering of light, which consists of generation and measurement of non-classical states of the optical field, has seen a rapid development [1]. Several schemes, which include the Fock filtering scheme of D'Ariano et al. [2], photon adding and displacing scheme of Dakna et al. [3] and the optical state truncation of Pegg et al. [4]-[7] have been proposed and studied.

The optical state truncation scheme, which is also referred to as the quantum scissors device (QSD), was originally proposed for preparing superposition of vacuum and one-photon state by truncating a coherent light, and it has been modified by Koniorczyk et al. [7] to generate superposition of vacuum, one-photon and two-photon states. The original QSD scheme is built by two beam splitters, one of which is fed by one-photon in one input port whereas the second port is left at vacuum. One of the output ports of this beam splitter is fed to the second beam splitter where it is mixed with the coherent light. The output modes of the second beam splitter are detected and the condition in which one photon is detected in one of the modes

$\ddagger$ E-mail: ozdemir@koryuw01.soken.ac.jp 
and none in the other mode corresponds to the conditional preparation of vacuum and one-photon states at the output of the first beam splitter. Paris [8] has modified this scheme by replacing each beam splitter by a Mach-Zehnder interferometer and proposed a fully interferometric correspondent of the QSD in which relative weights of the vacuum and single-photon states can be tuned by varying the internal phase-shift of the interferometers.

In a recent study [9], we have proposed an experimental scheme for the practical realization of the QSD scheme for state truncation taking into account the realistic description of single-photon-state generation and photon counting detectors. In this paper, that study is extended to include generation of arbitrary superposition of vacuum and one-photon states and to discuss the trade-off between the fidelity and relative weights of the vacuum and one-photon states in the superposition. The effect of detector efficiency is described and the rate of preparation of the desired state is discussed. This analysis goes beyond the analyses of [4, [5] by considering the realistic descriptions of photodetection and single-photon generation.

\section{Experimental scheme for qubit generation}

The device proposed is schematically depicted in Fig.1. This scheme is based on the ideas developed in [11, 12]. It consists of a parametric down conversion crystal as the single-photon source, conventional photon counters for conditional measurement and 50:50 beam splitters for generation of entangled photon number states (BS1) and for the mixing of coherent state with the entangled state (BS2). The overall input to the QSD scheme is

$$
\hat{\rho}_{\text {in } 1}=\hat{\rho}_{\left(a_{1}, c_{1}\right)} \otimes|0\rangle_{a_{2} a_{2}}\langle 0|\otimes| \alpha\rangle_{b_{3} b_{3}}\langle\alpha|
$$

where $|\alpha\rangle_{b_{3}}$ is the coherent state to be truncated to prepare the desired qubit state, and $\hat{\rho}_{\left(a_{1}, c_{1}\right)}$ is a mixed state density operator obtained at the output of the parametric down conversion after averaging over all possible phases and is given as

$$
\hat{\rho}_{\left(a_{1}, c_{1}\right)}=\left(1-\gamma^{2}\right)\left[|00\rangle\left\langle 00\left|+\gamma^{2}\right| 11\right\rangle\left\langle 11\left|+\gamma^{4}\right| 22\right\rangle\langle 22|+\cdots\right]_{\left(a_{1}, c_{1}\right)}
$$

where $\gamma^{2}$, typically $\sim 10^{-4}[10$, corresponds to the rate of one photon pair generation per pulse of the pump field. The action of a beam splitter (BS1 and BS2) is represented by a unitary operator $\hat{U}$. Given the input density operator $\hat{\rho_{1}}$ of a lossless $50: 50$ beam splitter, the output can be calculated using $\hat{U}^{\dagger} \hat{\rho}_{1} \hat{U}$. $\hat{U}$ is conventionally given by

$$
\hat{U}=\exp \left[-i\left(\psi_{t}-\psi_{r}\right) \hat{L}_{3}\right] \exp \left[-i 2 \theta \hat{L}_{2}\right] \exp \left[-i\left(\psi_{t}+\psi_{r}\right) \hat{L}_{3}\right]
$$

where

$$
\hat{L}_{2}=\frac{1}{2 i}\left(\hat{a}_{1}^{\dagger} \hat{a}_{2}-\hat{a}_{2}^{\dagger} \hat{a}_{1}\right), \quad \hat{L}_{3}=\frac{1}{2}\left(\hat{a}_{1}^{\dagger} \hat{a}_{1}-\hat{a}_{2}^{\dagger} \hat{a}_{2}\right),
$$

with $\theta=\pi / 4$. $\quad \psi_{t}=0$ and $\psi_{r}=\pi / 2$ correspond to the phases of the beamsplitter transmission and reflection parameters, respectively. After the action of the beam splitters (BS1 and BS2) on the input state $\hat{\rho}_{\text {in } 1}$, the output state before the photodetection process becomes

$$
\hat{\rho}_{\left(c_{1}, c_{2}, c_{3}, b_{1}\right)}=\hat{U}_{2}^{\dagger} \hat{U}_{1}^{\dagger}\left(\hat{\rho}_{\left(a_{1}, c_{1}\right)} \otimes|0\rangle_{a_{2} a_{2}}\langle 0|\otimes| \alpha\rangle_{b_{3} b_{3}}\langle\alpha|\right) \hat{U}_{1} \hat{U}_{2}
$$




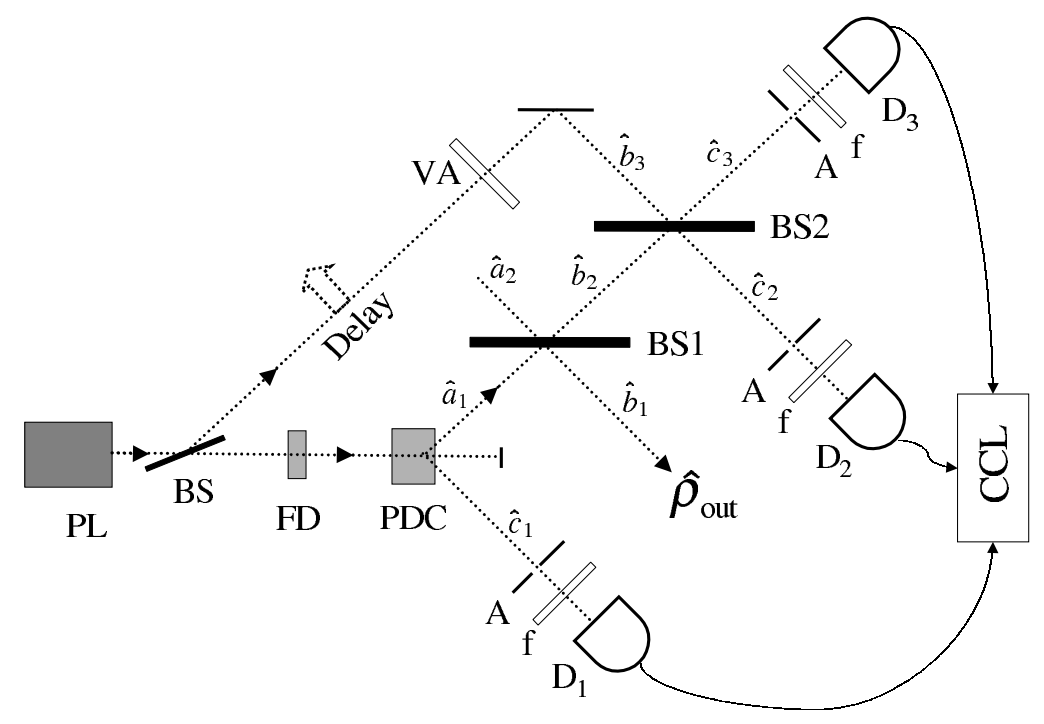

Figure 1. Experimental QSD scheme for state truncation and qubit state preparation: PL - pulsed laser; FD - frequency doubler; PDC - parametric down conversion crystal; VA - variable attenuator; A - aperture; $\mathrm{f}$ - narrow band filter; CCL - coincidence counter and logic; BS, BS1, and BS2 are beam splitters; and $\mathrm{D}_{1}, \mathrm{D}_{2}$, and $\mathrm{D}_{3}$ are photon-counting detectors.

The normalized truncated output state density operator at mode $\hat{b}_{1}$ of BS1 after the conditional measurement of coincidence detection ("click") at detectors D1 and D2 and anti-coincidence ("no-click") at D3 is obtained by

$$
\hat{\rho}_{\text {out }}=\frac{\operatorname{Tr}_{\left(c_{1}, c_{2}, c_{3}\right)}\left(\Pi_{1}^{c_{1}} \Pi_{1}^{c_{2}} \Pi_{0}^{c_{3}} \hat{\rho}_{\left(c_{1}, c_{2}, c_{3}, b_{1}\right)}\right)}{\operatorname{Tr}_{\left(b_{1}, c_{1}, c_{2}, c_{3}\right)}\left(\Pi_{1}^{c_{1}} \Pi_{1}^{c_{2}} \Pi_{0}^{c_{3}} \hat{\rho}_{\left(c_{1}, c_{2}, c_{3}, b_{1}\right)}\right)},
$$

where $\Pi_{1}^{c_{1}}, \Pi_{1}^{c_{2}}$, and $\Pi_{0}^{c_{3}}$ are elements of the positive-operator-valued measures (POVMs) for the detectors $\mathrm{D}_{1}, \mathrm{D}_{2}$, and $\mathrm{D}_{3}$, respectively, with 0 and 1 corresponding to the number of clicks recorded at the detectors. The measurement by conventional photon counters is described by the following two-value POVM

$$
\begin{aligned}
\Pi_{0} & =\sum_{m=0}^{\infty}(1-\eta)^{m}|m\rangle\langle m|, \\
\Pi_{N \geq 1} & =1-\Pi_{0} .
\end{aligned}
$$

with $\eta$ being the quantum efficiency of the detectors. The effect of mean dark count $\nu$ of the detector in the POVM has been neglected because in a previous study, it was shown that when $\nu \ll \gamma^{2}$, which can be achieved in experiments using commercially available detectors, dark count rate does not have a significant effect on the fidelity and efficiency of the truncation process.

Due to non-ideal detection and non-ideal single-photon generation (output of parametric down conversion process contains vacuum and higher number of photons together with a low rate of single-photon pair), the conditional output state $\hat{\rho}_{\text {out }}$ is not a pure state. However, as was shown in [9], there are regimes of intensity of the input coherent state and the detector parameters where the output state approaches the superposition state of vacuum and one-photon with non-vanishing 
probability. To compare $\hat{\rho}_{\text {out }}$ with the desired qubit state of the form $\left|\psi_{\text {desired }}\right\rangle=$ $N\left[c_{0}|0\rangle+c_{1}|1\rangle\right]$ with $N$ being the normalization constant, we consider the fidelity $F=\left\langle\psi_{\text {desired }}\left|\hat{\rho}_{\text {out }}\right| \psi_{\text {desired }}\right\rangle$.

\section{Preparation of qubit states with the experimental QSD scheme}

In the QSD scheme there are two kinds of free parameters, namely the intensity $|\alpha|^{2}$ of the coherent light and the beam splitter parameter (reflectance and transmittance), that can be tuned to properly set the relative weights of the vacuum and one-photon states in the superposition of $N\left[c_{0}|0\rangle+c_{1}|1\rangle\right]$. The tuning of the beam splitter parameters can be realized by using the interferometric scheme of Paris [8]. This scheme makes the setup more complicated and introduces the problem of controlling the stability and balance of the interferometers. The adjustment of the $|\alpha|^{2}$ can be realized without introducing additional complexity to the setup except a controllable tuning of the intensity of the coherent light. However, this may also constitute a challenge for the preparation of some specific qubit states which needs very fine tuning of the intensity.

In this study, our aim is to optimize the intensity $|\alpha|^{2}$ of the input coherent light to generate an arbitrary qubit state with the highest fidelity with non-vanishing probability of the state generation. In that case, $B S 1$ and $B S 2$ are chosen as $50: 50$ beam splitters because they give the highest probability and fidelity for state truncation using the ideal QSD scheme [4. 9]. The highest fidelity in generating arbitrary qubit states from a coherent state can be achieved if $\arg (\alpha)=\arg \left(c_{1}\right)$, which is assumed to be the case in this study.

In the following, the results of numerical simulations for the proposed experimental QSD scheme are presented. The simulations were performed for different $|\alpha|^{2}$ and $\eta$ using the POVM given by (7). Parametric down conversion output was used in the simulations in the form of (2) and $\gamma^{2}$ parameter was taken as $4 \times 10^{-4}$ for which the ratio of one-photon pair generation to that of the two-photon pair generation is $O\left(10^{4}\right)$ resulting in a low probability of having contributions from higher number of photons at the output mixed state.

\subsection{Equally weighted superposition of vacuum and one-photon states}

Effects of detection efficiency and the intensity of the coherent light on the preparation of the qubit state which consists of equally weighted vacuum and one-photon components of the form $[|0\rangle+|1\rangle] / \sqrt{2}$ are analyzed. The results of these simulations are depicted in figure 2. The relation between $\eta$ and $|\alpha|^{2}$ to achieve a given fidelity is clearly seen in this figure. For $|\alpha|^{2} \leq 0.36$, fidelity is less than 0.9 for any value of detector efficiency. At much smaller values of intensity, fidelity is not affected by the detector efficiency. At a constant $|\alpha|^{2}$, the number of correct detection events increases with increasing detector efficiency. It is seen that equally weighted superposition of vacuum and single-photon states can be prepared with high fidelity even for $\eta$ as low as 0.1 provided that the intensity of the coherent light is chosen properly. For $\eta=0.5$, the optimum value for $|\alpha|^{2}$ is 0.72 for which the fidelity of the prepared state to the desired one becomes 0.89 . Such a detection and state preparation can be achieved with a rate of $4533 \mathrm{~s}^{-1}$. For each detection efficiency, the optimized value of 

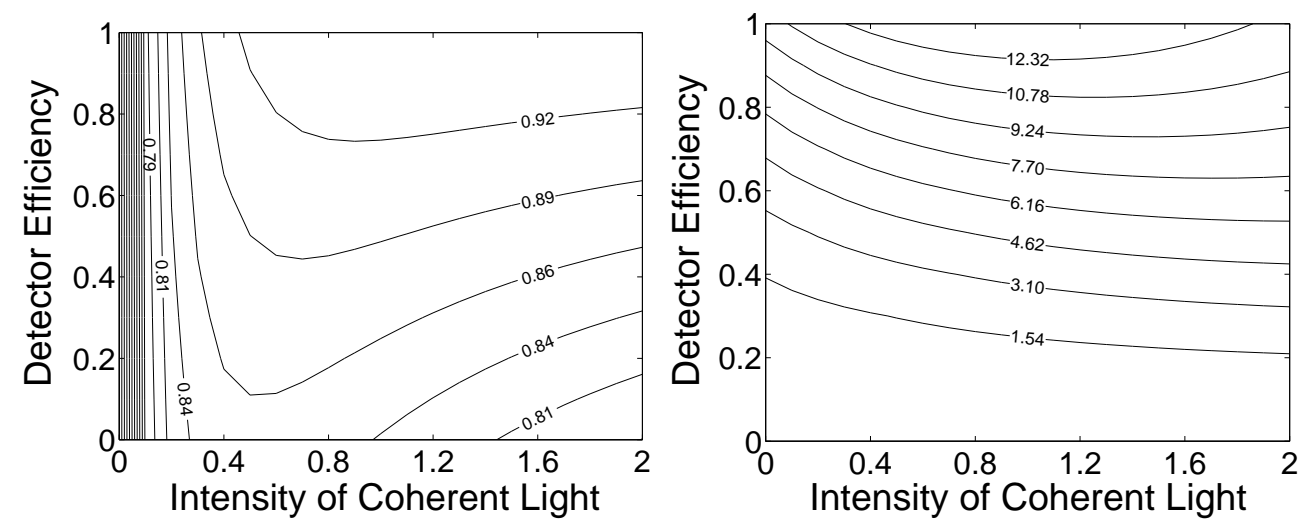

Figure 2. Effect of intensity of the input coherent light $|\alpha|^{2}$ and detector efficiency $\eta$ on the fidelity $F$ (left) and the rate $R$ of correct detection event (right) of the balanced superposition state generation. Constant $F$ and $R$ curves are depicted in the figure. $R$ curves are scaled with 100 for clarity.

$|\alpha|^{2}$ and the maximum attainable fidelity are different. Increasing $\eta$ from 0.5 to 0.7 will increase the value of $|\alpha|^{2}$ to 1.06 and will change the fidelity only by $0.11 \%$. On the other hand, the generation rate of such a state is almost doubled to a value of $8524 \mathrm{~s}^{-1}$.

\subsection{Arbitrary superposition of vacuum and one-photon states}

The intensity $|\alpha|^{2}$ of the coherent light has been optimized to give the maximum fidelity to prepare an arbitrary superposition of vacuum and one-photon state in the form of

$$
\left|\psi_{\text {desired }}\right\rangle=\frac{c_{0}|0\rangle+c_{1}|1\rangle}{\sqrt{\left|c_{0}\right|^{2}+\left|c_{1}\right|^{2}}}
$$

Figure 3 depicts the values of the optimized $|\alpha|^{2}$ and the maximum value of fidelity that can be achieved for any desired state of $\left|c_{1} / c_{0}\right|$ with that value of $|\alpha|^{2}$ for different detector efficiencies. It has been understood that an optimum $|\alpha|^{2}$, which will yield a maximum fidelity with non-zero preparation rate, can always be found for any arbitrary desired state. This optimum value of $|\alpha|^{2}$ depends on the detector efficiency $\eta$ and $\left|c_{1} / c_{0}\right|$. When $\left|c_{1} / c_{0}\right| \leq 0.4$, a fidelity value of $\sim 0.99$ can be achieved with the optimized $|\alpha|^{2}$ for $\eta \geq 0.5$. The fidelity of the qubit state preparation depends significantly on the relative weights of the vacuum and single-photon states in the superposition. If the vacuum component is dominant in the desired state, then high fidelity values greater than 0.9 can be achieved even for smaller detector efficiencies. But when the one-photon component becomes dominant, the fidelity of the state preparation starts decreasing. For $\left|c_{1} / c_{0}\right|=0.5, F \simeq 0.98$ at the optimized value $|\alpha|^{2} \simeq 0.21$ and $\eta=0.5$, however when the desired state is $\left|c_{1} / c_{0}\right|=2$, the fidelity drops to 0.74 at the optimized value of $|\alpha|^{2} \simeq 2.7$. If $\eta$ is increased, fidelity increases to only $\sim 0.77$ at $\eta=0.7$, and to $\sim 0.8$ at $\eta=0.9$. All of these arbitrary qubit states can be generated with non-zero preparation rate. This preparation rate increases with increase in $\left|c_{1} / c_{0}\right|$ up to some maximum value which depends on $\eta$ and then starts decreasing when one-photon state becomes more dominant in the superposition.

It is observed that when detector efficiency is in the range of $1.0 \leq \eta \leq 0.9$, for some values of $\left|c_{1} / c_{0}\right|$, two different values of optimum $|\alpha|^{2}$ at which fidelity takes the 
(a)

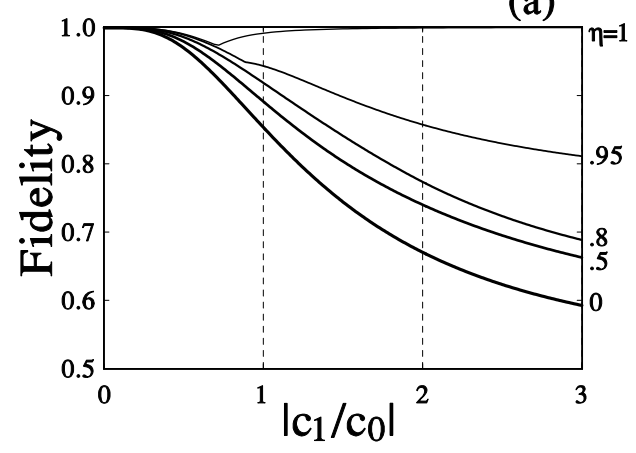

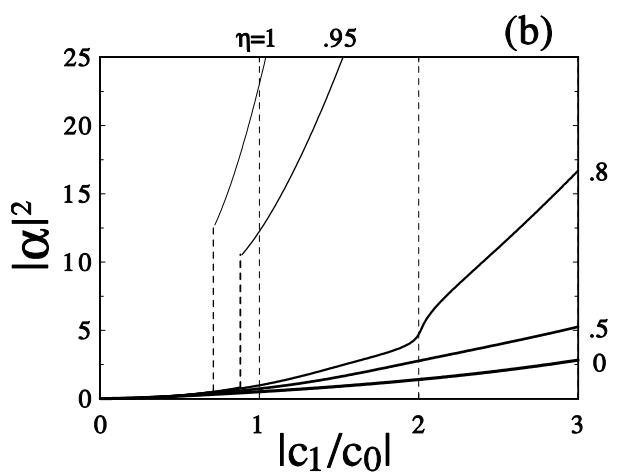

Figure 3. Optimized $|\alpha|^{2}$ for preparing qubit states of arbitrary $\left|c_{1} / c_{0}\right|$ and the corresponding maximum fidelity for various detector efficiencies.

same maximum value can be found (see figure 3(b)). Although fidelity reaches the maximum value for these two values of $|\alpha|^{2}$, the corresponding rates of the preparation of the state are different. As it can be seen in figure 3 , for the preparation of the qubit state with $\left|c_{1} / c_{0}\right| \simeq 1.145$ using detectors of $\eta=0.9$, the maximum fidelity of $\sim 0.906$ can be obtained at $|\alpha|^{2} \simeq 1.464$ and $|\alpha|^{2} \simeq 8.644$ for which the state preparation rates are $11869 \mathrm{~s}^{-1}$ and $561 \mathrm{~s}^{-1}$, respectively. In the same way, for $\left|c_{1} / c_{0}\right| \simeq 0.712$, if we increase $\eta$ to unity, the state preparation rate will become $13330 \mathrm{~s}^{-1}$ and $35 \mathrm{~s}^{-1}$ at $|\alpha|^{2} \simeq 0.548$ and $|\alpha|^{2} \simeq 12.532$, respectively, with the same maximum attainable fidelity of $\sim 0.973$.

It must be noted that optimizing the intensity of the coherent light to obtain the maximum fidelity for a desired qubit state does not necessarily mean that the system is maximized for the highest preparation rate, too. The cost of maximizing the fidelity is the decrease in the preparation rate and vice versa. At $\eta=0.7$, to prepare a qubit state having $\left|c_{1} / c_{0}\right|=0.4$ with the highest attainable fidelity of 0.992 , the optimum $|\alpha|^{2}=0.152$ must be used which will result in preparation rate of $5790 \mathrm{~s}^{-1}$. However, if the system is optimized to have the highest preparation rate, an optimum value of $|\alpha|^{2}=1.538$ needs to be used. This will result in a fidelity of 0.859 which is much lower than that of the former case. At $\eta \geq 0.7$, only for the case of balanced superposition state, the values of fidelity and state preparation rate obtained when the system is optimized for the highest fidelity are very close to those obtained when optimization is made for the highest preparation rate.

Figure 4 depicts the comparison of the ideal QSD scheme of Barnett et al. [5] and the proposed experimental scheme for $|\alpha|^{2} \leq 4$ and $\eta=0.5$. We have not considered the larger values of $|\alpha|^{2}$, because at these higher values of light intensity, even though in some cases, fidelity has a higher value, the state preparation rate approaches very small values which are not experimentally feasible. The comparison has been done in three different regions of $\left|c_{1} / c_{0}\right|$ for various $\eta$ : (a) For $\left|c_{1} / c_{0}\right|<1.0$, which means vacuum dominated qubit state, fidelity increases with increasing $|\alpha|^{2}$ until it reaches the maximum value for both schemes. After that maximum value, fidelity starts decreasing with respect to $|\alpha|^{2}$, however the experimental scheme overwhelms the results obtained in [5] for the ideal scheme and has much higher fidelity values. It is 
seen in figure 1 that for $\left|c_{1} / c_{0}\right|=0.4$, the fidelity values for both schemes are very close to each other until $|\alpha|^{2} \simeq 0.28$ beyond which fidelity of the ideal scheme decreases
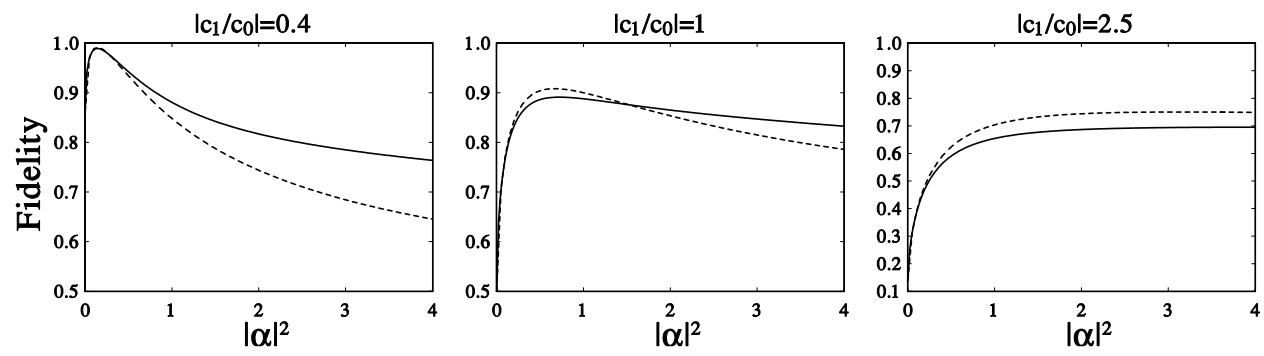

Figure 4. Comparison of the dependence of fidelity for the proposed experimental scheme and the ideal QSD scheme when they are used for preparing arbitrary qubit states. Solid line and dotted line correspond to experimental and the ideal QSD schemes, respectively.

more rapidly with increasing values of $|\alpha|^{2}$. (b) For the case of balanced superposition state having $\left|c_{1} / c_{0}\right|=1.0$, ideal QSD scheme has higher value of fidelity than the experimental scheme up to a critical value of $|\alpha|^{2} \simeq 1.52$ after which the experimental scheme starts to give slightly higher fidelity values; this difference is not as pronounced as the difference seen in case (a). (c) For $\left|c_{1} / c_{0}\right|>1.0$ corresponding to the onephoton-dominant qubit states, the ideal QSD scheme prepares states with higher fidelity than that of the proposed experimental scheme. When the total number of photons incident on the detectors is large, the output state approaches the vacuum state enhancing the advantage of the experimental scheme.

\section{CONCLUSIONS}

The experimentally feasible optical state truncation scheme proposed in [9] has been analyzed for the preparation of arbitrary qubit states of the form given by (8). State preparation is based on truncating a coherent light to contain only its vacuum and one-photon components. The relative weights of the vacuum and one-photon states in the qubit state can be adjusted by manipulating the intensity of the coherent light. It is understood that the qubit states, which are dominated by the vacuum component, can be prepared with high fidelity and efficiency with the proposed scheme considering the realistic descriptions of photodetection and single-photon generation. Moreover, one can always find an optimum value for the intensity of the coherent light which will maximize the fidelity. However, the price for the maximization of the fidelity is the decrease in the preparation rate of the state. This study reveals that the original QSD scheme and the proposed experimental scheme can be used not only for truncating an input coherent state but also for generating arbitrary qubit states with proper manipulation of the light intensity.

\section{Acknowledgments}

We thank Prof. Stephen M. Barnett for pointing us this problem and for his invitation and hospitality during Quantum Electronics and Photonics 15 (QEP-15) conference. 
We also thank Takashi Yamamoto and Yu-xi Liu for their stimulating discussions. This work was supported by a Grant-in-Aid for Encouragement of Young Scientists (Grant No. 12740243) and a Grant-in-Aid for Scientific Research (B) (Grant No. 12440111) by Japan Society for the Promotion of Science.

\section{References}

[1] Feature issue on Quantum State Preparation and Measurement, 1997, J. Mod. Opt. 44 (11/12).

[2] D’Ariano, G. M., Maccone, L., Paris, M. G. A., and Sacchi, M. F., 2000, Phys. Rev. A, 61, 053817.

[3] Dakna, M., Clausen, J., Knöll, L., and Welsch, D. G., 1999, Phys. Rev. A, 59, 1658.

[4] Pegg, D. T., Phillips, L. S., and Barnett, S. M., 1998, Phys. Rev. Lett., 81, 1604.

[5] Barnett, S. M., and PegG, D. T., 1999, Phys. Rev. A, 60, 4965.

[6] Villas-Bas, C. J., de Almeida N. G., and Moussa, M. H. Y., 1999, Phys. Rev. A, 60, 2759.

[7] Koniorczyk, M., Kurucz, Z., Gábris, A. and J. Janszky, 2000, Phys. Rev. A, 62, 013802.

[8] Paris, M. G. A., 2000, Phys. Rev. A, 62, 033813.

[9] Özdemir. S. K.. Miranowicz, A., Konshi, M., and Iмото, N., 2001, Phys. Rev A, 64, 063818; quant-ph/0107048.

[10] Bouwmeester, D., Pan, J.-W., Weinfurter, H., and A. Zeilinger, 2000, J. Mod. Opt., 47, 279.

[11] Rarity, J. G., and Tapster, P. R., 1999, Phys. Rev. A. 59. R35.

[12] Rarity, J. G., TAPSter, P. R. and Loudon, R., quant-ph/9702032.

to appear in the Journal of Modern Optics (spring 2002). 